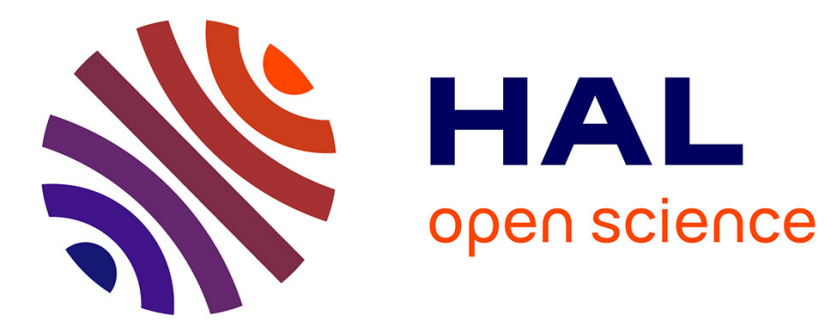

\title{
Les apports archéologiques des gravures rupestres de l'Aïr (Niger) et de l'Adrar des Iforas (Mali)
}

\author{
Christian Dupuy
}

\section{To cite this version:}

Christian Dupuy. Les apports archéologiques des gravures rupestres de l'Aïr (Niger) et de l'Adrar des Iforas (Mali). Les Nouvelles de l'archéologie, 2010, 120-121, pp.29-37. halshs-00686784

\section{HAL Id: halshs-00686784 https://shs.hal.science/halshs-00686784}

Submitted on 11 Apr 2012

HAL is a multi-disciplinary open access archive for the deposit and dissemination of scientific research documents, whether they are published or not. The documents may come from teaching and research institutions in France or abroad, or from public or private research centers.
L'archive ouverte pluridisciplinaire HAL, est destinée au dépôt et à la diffusion de documents scientifiques de niveau recherche, publiés ou non, émanant des établissements d'enseignement et de recherche français ou étrangers, des laboratoires publics ou privés. 


\section{Les apports archéologiques des gravures rupestres de l'Aïr (Niger) et de l'Adrar des Iforas (Mali)}

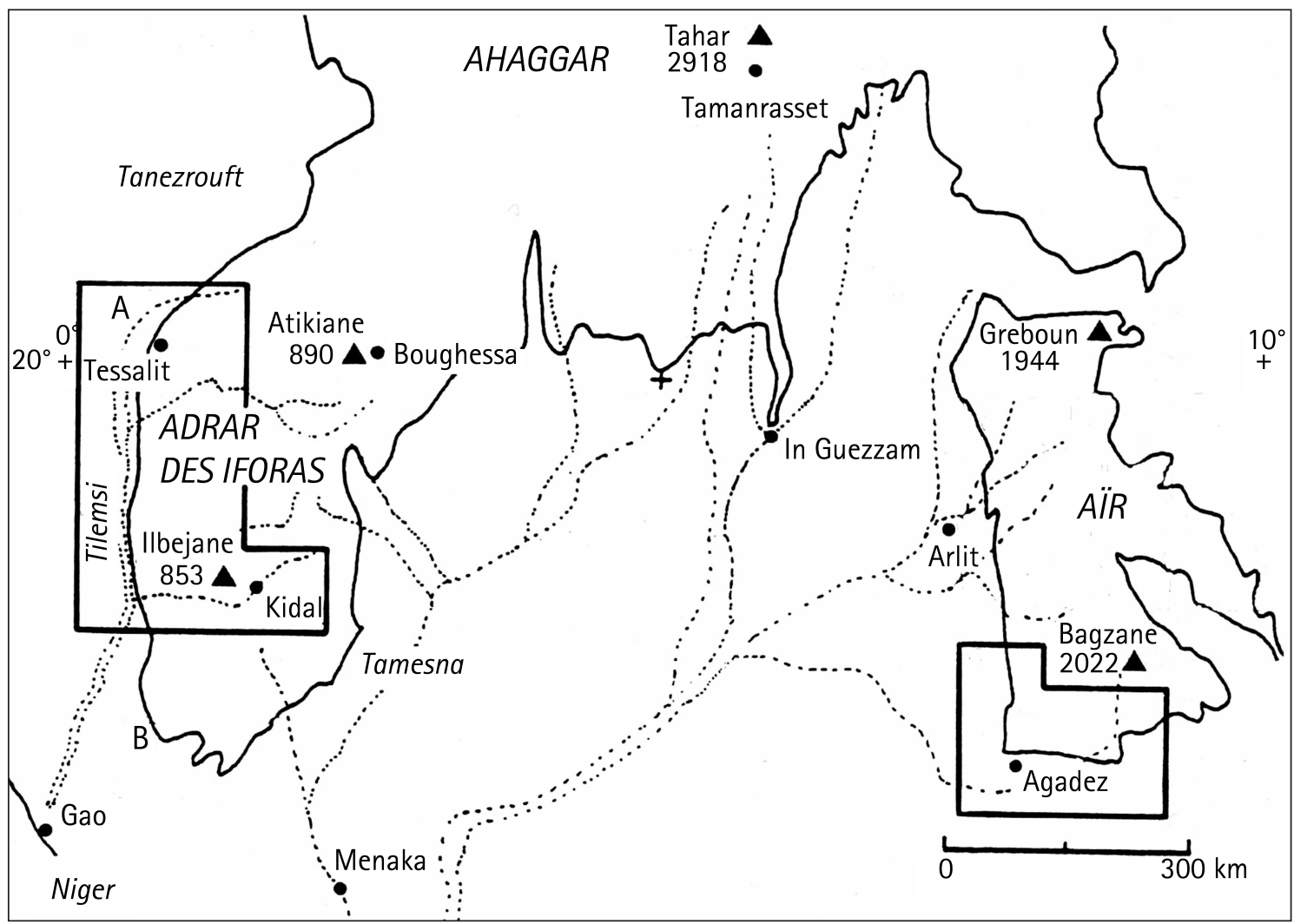

Fig. 1 - Encadrés : secteurs prospectés de 1983 à 1985 dans l'Aïr (Niger), puis en 1986 et 1990 dans l'Adrar des Iforas (Mali)

Historique et contexte des recherches

* UMR 5060 CNRS, Belfort;

Universités tous âges, Lyon, dupuy.family-christian@wanadoo.fr.
Darti en 1983 enseigner à l'École des mines de l'Aïr à Agadez au titre de la coopération militaire, j'ai étudié pendant deux ans l'art rupestre du sud-ouest de l'Aïr avec l'accord du ministère de l'Enseignement supérieur nigérien. J'ai concentré mon attention sur trois sites particulièrement riches en gravures et inscriptions alphabétiques que j'ai relevées de manière systématique avant de les analyser par ensembles, complétant ainsi les données enregistrées entre 1977 et 1981 au cours du Programme archéologique d'urgence d'In Gall et Tegidda $n$ Tesemt. Le classement en chronologie relative de ces expressions sur la base des styles, des thèmes et des superpositions, posait en des termes nouveaux les questions de l'origine, des traditions culturelles et du devenir des graveurs qui les avaient réalisées (Dupuy 1988). Ces résultats m'ont amené à poursuivre mes recherches dans l'Adrar des Iforas (Mali), un massif granitique de basse altitude situé à peu près aux mêmes latitudes que l'Aïr (fig. 1). L'Institut des sciences humaines de Bamako a appuyé ce projet complémentaire au Programme d'inventaire des sites archéologiques entrepris plus au sud. J'ai donc prospecté pendant trois mois en 1986, puis réalisé deux autres missions en 1990, la première 

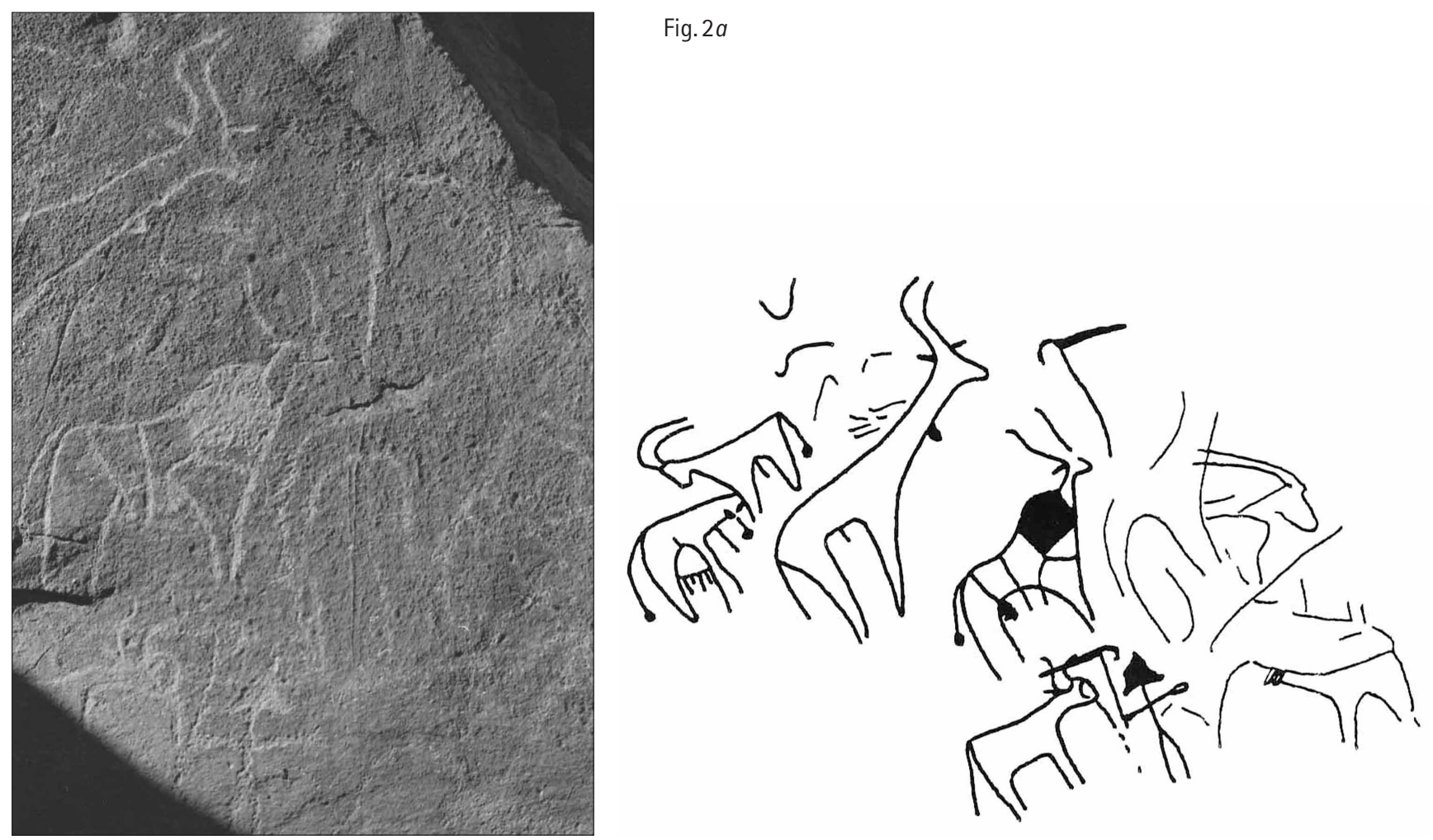

financée par le ministère de la Coopération française, la seconde, au sud de l'Adrar des Iforas sur le site de Taouardeï, à l'invitation du Centro Studi Archeologia Africana de Milan.

Mes recherches se concentrent dans six vallées successives du versant nord occidental où les prospections révèlent 34 stations de gravures. Toutes sont localisées avec précision grâce aux photographies aériennes au 1/50 000 de l'Institut géographique national. La position topographique de chaque paroi ornée est reportée sur un plan de situation. Les gravures qui ne peuvent être photographiées par manque de recul sont décalquées au feutre sur des films transparents posés à même les rochers. Le travail de laboratoire consiste à reproduire à l'encre au 1/20 les quelque 8000 motifs relevés, en plaçant les négatifs dans un agrandisseur photographique et les transparents sur des quadrillages aux mailles de dimensions appropriées. Les milliers de dessins sont rassemblés dans un corpus. Des bourses de la Fondation Fyssen et de la Fondation de France me permettent d'exploiter cette riche documentation. L'étude, station par station, des motifs et des thèmes traités sur les rochers, de la structure des compositions, des styles, des figures liées par superpositions et, simultanément, des attitudes des personnages représentés, de leurs vêtements, parures, armes et autres éléments associés de la culture matérielle, aboutit à la reconnaissance d'expressions distinctes et à une caractérisation fine de chacune d'elles. Ces données révèlent de nombreuses affinités avec celles des régions voisines et par là même l'existence d'aires culturelles. Quelques bribes de l'histoire du peuplement sud-saharien sont restituées par la mise en regard de ces résultats avec ceux enregistrés dans les domaines de la paléo- écologie, de la préhistoire, de la protohistoire, de l'histoire et de l'ethnologie de l'Afrique septentrionale (Dupuy 1991).

\section{Bilan scientifique}

Des éleveurs mobiles au Néolithique ancien

Le versant nord occidental de l'Adrar des Iforas recèle une cinquantaine de représentations humaines et animales que singularise leur allure dynamique due à un traitement élaboré des membres. Aucune gravure de ce style n'a été répertoriée jusqu'à présent dans l'Aïr. Par commodité, elles sont qualifiées de "naturalistes" pour les différencier des milliers d'autres représentations du Sahara méridional qui, soumises à de nombreuses stylisations, coupent court à toute animation.

Les taurins (Bos taurus ou bovinés domestiques à dos droit) aux robes parfois compartimentées et aux cornes variées arrivent en tête des sujets gravés. Viennent ensuite, par ordre décroissant d'importance, les éléphants, les girafes, deux personnages, deux lionnes, deux autruches, deux rhinocéros blancs, un rhinocéros noir, une antilope guib. À cette époque, les taurins étaient à un stade de domestication avancé comme l'atteste la diversité de leur robe et de leurs cornes, et la pluviosité était suffisante pour la survie de la grande faune sauvage.

Six gravures naturalistes de taurins et onze d'animaux sauvages sont sous-jacentes à d'autres plus schématiques renvoyant à des thèmes différents. Les premières présentent souvent des patines plus foncées que les secondes. L'ordre inverse de recouvrement ne s'observe sur aucune paroi; ce 
Fig. 2

$a$ (page de gauche) - Bœuf à bosse entouré d'un objet coudé traité isolément et d'un autre brandi à bout de bras par un personnage de petite taille (In Tahaten).

$b$ (à droite) - Char à timon unique figuré à côté de deux objets coudés (Tirist).

Il y a tout lieu de penser que les gravures réunies

sur chacune de ces parois, qui présentent la même patine et qui furent réalisées selon la même technique du piquage, sont contemporaines.

qui suppose que les auteurs des gravures naturalistes furent les premiers à s'exprimer dans l'Adrar des Iforas avec des préoccupations différentes de celles des graveurs qui, à des époques plus récentes, se remirent à inciser les granites de la région. Quelques représentations au naturalisme mal affirmé sont gravées aux côtés de ces figures naturalistes. Malgré leur différence de style, leur composition témoigne de leur contemporanéité. Aussi convient-il de parler ici d'un art rupestre à figures naturalistes plutôt que d'un art purement naturaliste (Dupuy 1999).

Plus de 15000 gravures renvoyant par leurs styles et leurs thèmes à celles de l'Adrar des Iforas ont été relevées surtout dans les Messaks libyens et les Tassilis algériennes, mais aussi dans l'Aramat, la Tadrart, l'Ahaggar, le Djado et sur le versant occidental du Tibesti. Les taurins occupent en tous lieux une place éminente, suivis par les animaux de la grande faune sauvage. Vient ensuite un éventail de motifs figuratifs et abstraits d'étendue variable suivant les régions.

Certaines parois ornées nous renseignent sur le vêtement, la parure et l'armement, quelquefois sur les pratiques pastorales telles que la traite des vaches, le harnachement de bœufs porteurs, la mise à mort de taurins... D'autres, en revanche, s'écartent de la réalité comme ces êtres composites à tête et denture de carnivore et corps d'humain marchant à paisible allure avec un aurochs posé sur les épaules et une tête de rhinocéros pendue à la ceinture. Ailleurs, ces mêmes personnages fabuleux, munis d'une hache, d'un couteau ou d'une

matraque, affrontent en toute quiétude des pachydermes. Ça et là, des anthropomorphes au pénis disproportionné ont des relations sexuelles avec des personnages féminins ou avec des animaux sauvages.

La pratique de l'élevage des taurins, des chèvres et des moutons est attestée à partir de la fin du $\mathrm{VI}^{\mathrm{e}}$ millénaire avant J.-C. On sait d'autre part, grâce aux données des sciences de la terre, que l'aridité s'est intensifiée dans le Sahara libyco-égyptien à partir du VII ${ }^{\mathrm{e}}$ millénaire avant J.-C. À l'aube du III millénaire avant J.-C., elle était, semble-t-il, à tel point marquée au nord du Tropique du Cancer, que les hippopotames et les rhinocéros blancs que l'on retrouve gravés dans les Messaks libyens ne pouvaient y survivre. Les connaissances archéozoologiques et paléoclimatiques actuelles invitent ainsi à dater l'art à gravures naturalistes 
entre le $\mathrm{VI}^{\mathrm{e}}$ et la fin du IV $\mathrm{IV}^{\mathrm{e}}$ millénaires avant J.-C. sans que l'on puisse préciser sa durée exacte d'expression, ni ses lieux de naissance et de disparition.

Alors que l'aridité du Sahara du Nord allait croissante, les bassins hydrographiques des fleuves Niger et Sénégal et du lac Tchad restaient couverts d'étendues d'eau pérennes grâce à des pluies de mousson plus abondantes qu'aujourd'hui. Cette humidité variable suivant la latitude a vraisemblablement encouragé les auteurs de l'art rupestre à gravures naturalistes à se déplacer au gré des saisons pour satisfaire les besoins en eau et en pâturage de leurs animaux. Certains d'entre eux atteignirent l'Adrar des Iforas où l'on dénombre une cinquantaine d'œuvres naturalistes. Aussi marginales soient-elles, ces gravures constituent les premiers indices d'une pratique de l'élevage au nord-est du Mali entre le $\mathrm{VI}^{\mathrm{e}}$ et la fin du IV ${ }^{\text {e }}$ millénaires avant J.-C. À la suite d'une forte mobilité peut-être imposée par le biotope, les structures de cette société du Néolithique ancien éclatèrent.
Des interactions à grande distance au lle millénaire avant J.-C. Désormais, quelques droites et courbes incisées suffisent aux graveurs pour délimiter les silhouettes d'animaux sans perspective ni attitude caractéristique. Cet art schématique s'exprime dans l'Adrar des Iforas et dans l'Aïr. Le bestiaire est dominé par les taurins, puis par les autruches et les girafes. Du mufle de ces dernières est parfois dessiné un trait jusqu'à la main ou sur la tête de petits personnages. En l'absence d'humain, ce trait se referme sur leur cou ou est laissé flottant. Des signes curvilignes s'intègrent dans ce contexte (Dupuy 1994). Quelques-uns se greffent sur les silhouettes de certains animaux. Les figures humaines sont partout faiblement représentées, leur style est épuré et leur taille toujours inférieure à 30 centimètres. Leurs coiffures, parures ou vêtements ne sont pas détaillés. Leurs armes sont des arcs au bois plus ou moins flexueux ou des objets coudés : les exemplaires gravés dans l'Aïr s'apparentent à de simples crosses tandis que
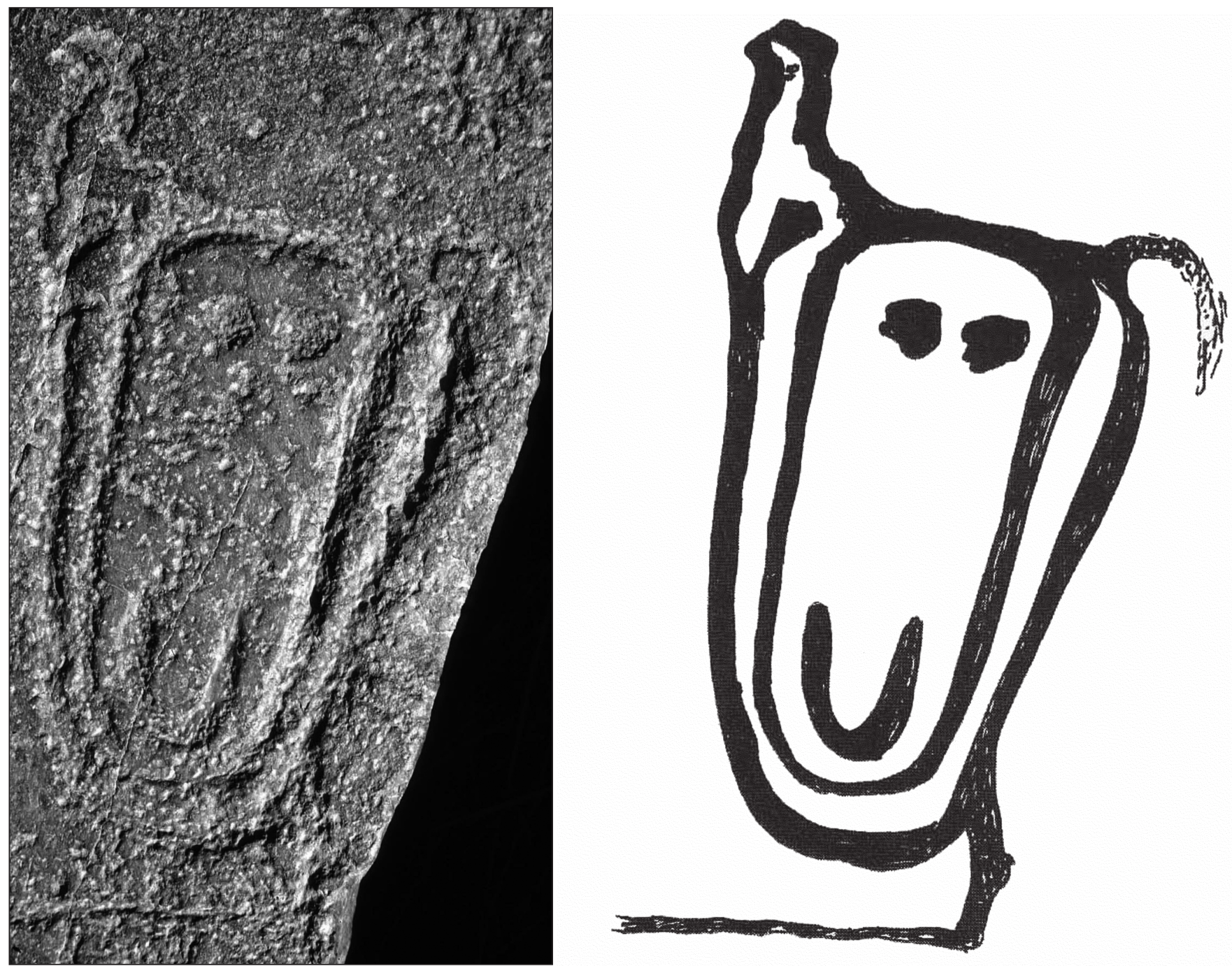

Fig. 3 - Ovale à double ponctuation gravé à Issamadanen dans I'Adrar des Iforas (hauteur : $30 \mathrm{~cm}$ ) 
Fig. 4 - Motif à quatre tentacules et neuf cupules gravé à Issamadanen dans I'Adrar des Iforas (hauteur : $40 \mathrm{~cm}$ )

ceux dessinés dans l'Adrar des Iforas ont des formes complexes. Ils apparaissent soit isolés ou groupés par paire ou par trois, soit brandis à bout de bras par des humains minimisés. Les lames sont pourvues d'un crochet à leur base. Leurs profils variés - foliacé, triangulaire, en croissant ou en demi-lune - suggèrent l'emploi d'un métal. Il n'y a que l'Adrar des Iforas qui ait livré à ce jour de telles figures. En effet, aucun objet métallique n'a été découvert jusqu'ici dans l'Aïr, ni plus à l'est à Termit et Dibella, ni plus au nord, dans les Tassilis algériennes, l'Ahnet et l'Ahaggar. Cet art rupestre peut être daté du Néolithique final et celui qui leur est apparenté dans l'Adrar des Iforas, des débuts de l'âge des métaux.

Deux parois ornées dans l'Adrar des Iforas conduisent à s'interroger sur l'époque à laquelle s'opère cette transition : l'une associe deux objets coudés à un char dételé, l'autre deux objets coudés à un bœuf à bosse (fig. 2). Ces figures n'ont pu être réalisées qu'après le $\mathrm{XVI}^{\mathrm{e}}$ siècle avant J.-C., époque où furent introduits dans la vallée du Nil les premiers chars et zébus en provenance du Proche-Orient (Dupuy 2005). Selon que l'on postule une diffusion lente ou rapide de ces objets de l'Égypte vers la moyenne vallée du Niger, on peut dater leur figuration et celle des objets métalliques associés entre le $\mathrm{II}^{\mathrm{e}}$ millénaire avant J.-C et les $\mathrm{IV}^{\mathrm{e}}-\mathrm{V}^{\mathrm{e}}$ siècles après J.-C., quand les premières représentations de dromadaires montés oblitèrent à de multiples endroits les expressions schématiques intégrant des objets coudés.

Un petit ensemble de motifs marginaux dans l'Adrar des Iforas plaide en faveur d'une transmission rapide : huit chars reliés par paire l'un devant l'autre, deux spirales développées en méandres et deux cruciformes. De tels motifs se retrouvent gravés et parfois peints à l'unité dans les massifs du Sahara central et sur le versant méridional de l'Atlas nord africain (Dupuy 2006). Leur spécificité, leur rareté et leur vaste répartition géographique permettent d'y voir les manifestations d'interactions rapides et conjuguées sur de longues distances. Une autre gravure de l'Adrar des Iforas conforte cette hypothèse : un ovale à double ponctuation imbriqué dans un $\mathrm{U}$ dont la branche montante gauche se termine en croissant (fig. 3). Cette excroissance évoque le profil des "haches peltes" gravées dans le Grand Atlas marocain et sur son piémont méridional, aux côtés de hallebardes, de poignards et de pointes à soie caractéristiques du Bronze ancien ibérique daté de la première moitié du II ${ }^{\mathrm{e}}$ millénaire avant J.-C. (Chenorkian 1988; Rodrigue 1999). Si cette identification est correcte, l'ovale gravé dans l'Adrar des Iforas représenterait alors un

anthropomorphe armé : les deux ponctuations en partie haute rendraient compte de ses yeux, l'arceau en partie basse soulignerait sa bouche tandis que le U symboliserait ses deux bras dressés avec une hache pelte tenue dans la main gauche. Des dizaines d'ovales à double ponctuation apparentés à ce motif dans l'Adrar des Iforas, ainsi que ceux dessinés dans des régions plus septentrionales, pourraient, eux aussi, représenter des anthropomorphes. Leur ressemblance avec les "idoles à tête de chouette" peintes, gravées et sculptées dans les pays européens de la Méditerranée occidentale au cours d'une période allant du Néolithique final à l'âge du Bronze (Abelanet 1986) est troublante. L'existence d'interactions à grande distance à travers le Sahara du II ${ }^{\mathrm{e}}$ millénaire avant J.-C. est d'autant plus envisageable que les représentations de chars, de bœufs à bosse et de motifs complexes présentés plus haut plaident déjà en ce sens. Un autre motif de l'Adrar des Iforas composé de quatre tentacules rayonnants associés à neuf cupules renforce cette hypothèse : une cupule est au centre, quatre autres sont disposées à équidistance entre les tentacules, les quatre restantes en marquent les extrémités (fig. 4). Ce dessin est identique aux rosa camuna relevées dans le Valcamonica (Lombardie) au voisinage d'«idoles à tête de chouette", de hallebardes et de couteaux caractéristiques du Bronze ancien italique. La présence de ces gravures ô combien particulières de part et d'autre de la Méditerranée ne peut s'expliquer que par des relations intercontinentales. Reste à saisir les modalités de ces transmissions : diffusion par contacts entre communautés voisines, mobilité généralisée par l'aridité du Sahara, déplacements de groupes de pasteurs nomades ou de quelques individus - colporteurs, prédicateurs, explorateurs... La documentation archéologique actuelle 


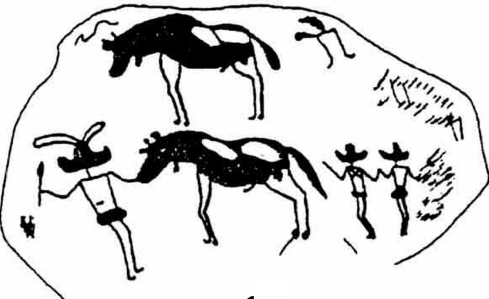

1

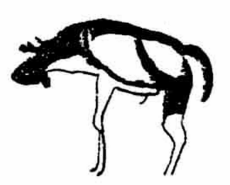

2

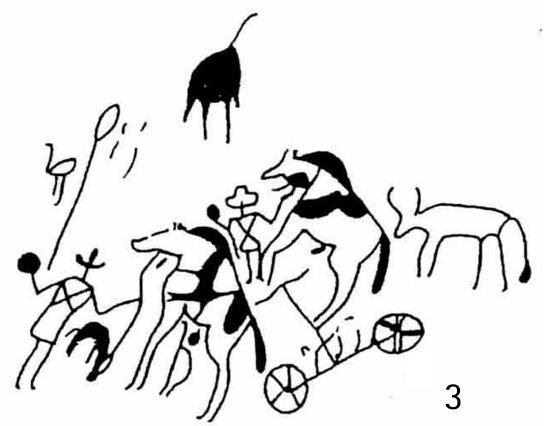

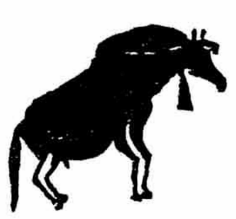

4
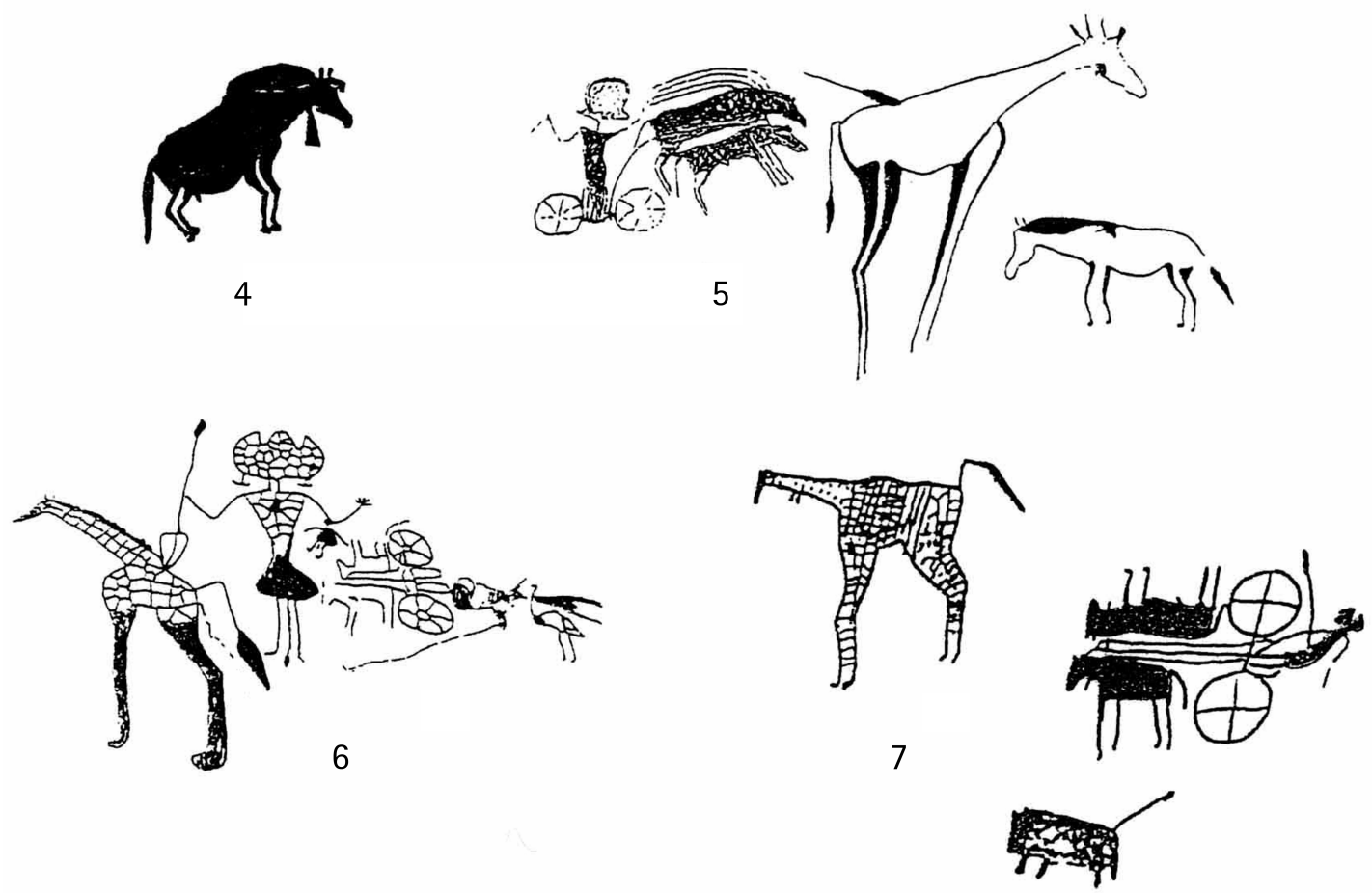

Fig. 5 - Les premières représentations gravées de chevaux dans l'Adrar des Iforas

(1 et 2 - Issamadanen; 3 - Asenkafa) et dans l'Aïr (4 et 7 - Tagueï ; 5 - Emouroudou; 6 - Iwelen)

ne permet pas de se prononcer. Quoi qu'il en soit, les divers éléments présentés s'accordent pour dater du $\mathrm{II}^{\mathrm{e}}$ millénaire avant J.-C. les premières représentations d'objets métalliques dans l'Adrar des Iforas. L'originalité de leur forme plaide en faveur d'une fabrication locale. Il appartient aux fouilles archéologiques à venir d'en retrouver les vestiges. Par ailleurs, la circulation de ces premiers objets en métal aux côtés de chars et de bœufs à bosse a dû attiser les convoitises, par le prestige à les posséder et, de fait, accentuer des inégalités sociales qu'il y avait.

\section{L'affirmation d'une société aristocratique au ler millénaire avant J.-C.}

Certaines gravures de l'époque des objets coudés sont oblitérées par des personnages traités de face. L'ordre inverse de superposition ne s'observe jamais. Dans l'Adrar des Iforas comme dans l'Aïr, ces figures humaines, en moyenne trois fois plus grandes que les précédentes, se comptent par centaines et les deux tiers sont clairement de sexe masculin (Dupuy 1988 et 1991; Lhote 1972, 1979, 1987). Les coiffures, coiffes, parures et vêtements étonnent par leur diversité. La lance à large pointe métallique, souvent renforcée d'une nervure centrale, constitue l'arme de prédilection. Le thème ignoré jusque-là de la domination des humains sur la grande faune sauvage participe de ce tournant : çà et là, des hommes fortement sexués appliquent la pointe de leur lance sur des éléphants, des rhinocéros ou des girafes minimisés. Quelques taurins sont ainsi aussi menacés. Des étalons, nouveaux dans le répertoire, sont représentés avec des porteurs de lance. Six d'entre eux sont attelés par paire à des chars (fig. 5). 
Le cheval a besoin de céréales pour fournir des efforts soutenus, ce qui nécessite de disposer d'importantes réserves de grains ou de pouvoir s'approvisionner, au besoin lance à la main, dans des greniers à mil et à sorgho de la région. Par ailleurs, le cheval est très vulnérable aux parasites et aux piqûres des mouches tsé-tsé. Conscients du problème, les Khassonkés, agriculteurs et éleveurs actuels de la haute vallée du Sénégal, abritent leurs montures dans des cases qu'ils enfument quotidiennement pendant les pluies de la mousson pour en chasser la vermine. Pour limiter les risques d'épizooties à la même saison, les Marbas, agriculteurs sédentaires du sud du lac Tchad, enferment leurs chevaux dans des écuries intégrées à l'habitat (Seignobos et al. 1987). Ces dispositions particulières montrent que le cheval ne peut s'accommoder d'une vie itinérante à longueur d'année en région tropicale. Aucun Peul nomade, éleveur de bovins de l'Ouest africain, n'élève de chevaux, à l'inverse des Peuls sédentaires établis dans les bassins du Niger et du Sénégal et autour du lac Tchad. Le cheval est donc source fréquente d'immobilité. Leurs premières représentations en Adrar des Iforas et en Aïr aux côtés d'animaux de la grande faune sauvage supposent donc un pastoralisme peu mobile, au moins durant les pluies de la mousson, de la part de personnages en position sociale dominante qui attelaient cet animal à des chars pour parfaire la stratégie de prestige de leur communauté.

Les résultats des fouilles de Jean-Pierre Roset (2007) et François Paris (1990) à Iwelen, au nord-est de l'Aïr, fixent le cadre chronologique de cette évolution. Trois pointes de lance en cuivre, découvertes dans une zone d'habitat occupée durant le $\mathrm{I}^{\mathrm{er}}$ millénaire avant J.-C, sont identiques à celles gravées alentours dans les mains de personnages représentés de face selon des conventions appliquées dans l'Aïr et dans l'Adrar des Iforas. Les multiples affinités entre l'art rupestre de ces deux massifs voisins du Sahara méridional, riches en figures de porteurs de lance, engagent à le dater du I ${ }^{\text {er }}$ millénaire avant J.-C. Ce témoin d'une hiérarchisation de la société s'accorde plutôt avec ce que l'on sait aujourd'hui de l'organisation politique et des stratégies de défense en œuvre dans différentes régions ouest-africaines au cours de cette période. La remarquable statuaire de terre cuite de la culture Nok du centre du Nigeria dénote pour lors un art savant, sinon déjà un art de cour, de la part de groupes établis sur des hauteurs parfois protégées de remparts de pierre (Boullier et al. 20022003 ; Rupp, Ameje \&t Breunig 2005). À 500 kilomètres de là, au sud-ouest du lac Tchad, à partir du v ve siècle avant J.-C., de larges et profonds fossés peuvent être associés à de puissants murs de terre creusés autour d'habitations rassemblées sur des dizaines d'hectares, attestant un même souci de protection (Magnavita et al. 2009). L'apparition d'éperons barrés et de villages ceints de murailles au sommet et sur les pentes des Dhars Tichitt et Oualata, longue falaise du Sud mauritanien, remonte au II ${ }^{\mathrm{e}}$ millénaire avant J.-C. (Amblard-Pison 2006). Dès le III $^{\mathrm{e}}$ siècle avant J.-C., à seulement 500 kilomètres de l'Adrar des Iforas, des agriculteurs se sont installés sur les levées alluviales de la moyenne vallée du Niger naturellement protégées par les eaux des crues pendant que d'autres construisaient des dizaines de greniers en boudins de glaise superposés dans une grotte perchée de la falaise de Bandiagara (Bedaux 1972; Bedaux et al. 1978; McIntosh \&t McIntosh 1980). Ces données, si éparses soient-elles, encouragent à approfondir les recherches sur la période des chars africains. Le scénario souvent avancé de migration-conquête de populations septentrionales s'avérera peut être trop réducteur et trop orienté, dès lors que l'on disposera de vestiges archéologiques en quantité suffisante pour des comparaisons interrégionales.

La mise en place des Touaregs

À l'art schématique des deux derniers millénaires avant J.-C. succède dans l'Aïr et l'Adrar des Iforas, sans transition thématique, un art au caractère narratif parfois marqué. Les animaux préférés sont désormais les chevaux et les dromadaires. Les compositions renvoient à des traditions prisées par les Touaregs : chasse à courre, port de javelots et d'habits bien couvrants, utilisation d'une écriture composée de signes très semblables aux tifinagh dont se servent ces pasteurs nomades pour transcrire quelques messages dans leur langue berbère. Ces thèmes et ces inscriptions se répartissent sur la majeure partie de leur domaine. Aussi est-il logique d'attribuer ces expressions à leurs ancêtres.

L'écriture, le port de javelots et la chasse à courre sont trois traditions apparues en Afrique du Nord au cours du I ${ }^{\mathrm{er}}$ millénaire avant J.-C. Des stèles découvertes dans des tumulus à chapelle de la région de Djorf Torba (piémont méridional de l'Atlas sud-oranais d'Algérie) montrent des hommes armés de plusieurs javelots dans des attitudes identiques à celles des guerriers de l'Adrar des Iforas et de l'Aïr. Les décors géométriques de certaines stèles et la figuration de croix latines ont conduit Gabriel Camps (1995) à les considérer comme contemporaines des derniers siècles de l'occupation romaine, époque où le dressage du dromadaire comme méhari se généralisa dans le Sahara du Nord. Ainsi, à partir des $\mathrm{IV}^{\mathrm{e}}$ et $\mathrm{V}^{\mathrm{e}}$ siècles après J.-C., des cavaliers et méharistes se sont rendus maîtres de territoires sahariens de plus en plus méridionaux où ils ont gravé et parfois peint des éléments propres à leurs manières de vivre, aujourd'hui encore spécifiques aux Touaregs (fig.6).

Cette mise en place des Touaregs, fortement suggérée par l'art rupestre, est documentée depuis peu par les fouilles. Trois tombes étudiées dans l'Aïr et ses environs par François Paris (1996) ont livré un matériel de facture manifestement berbère. La première est un tumulus à cratère édifié sur une plateforme gravillonnée, sous lequel était inhumée une femme parée d'un anneau en bronze à chaque cheville et d'un bracelet en corne à chaque bras, coiffée d'un voile de coton et vêtue d'une tunique en laine dont les motifs et la technique de tissage témoignent d'une influence, sinon d'une origine, septentrionale. Les datations donnent pour cette sépulture un âge moyen compris entre 780 et 945 après J.-C. La deuxième est une bazina à alignement datée sur fragments osseux de 890-1025 après J.-C. Un petit bol en terre cuite à fond conique et à embase plate, pourvu d'une oreille en partie haute, était placé dans la chambre funéraire. Son décor d'incisions parallèles croisées en losanges évoque les motifs rectilinéaires de la poterie berbère de l'Afrique du Nord protohistorique et histo- 

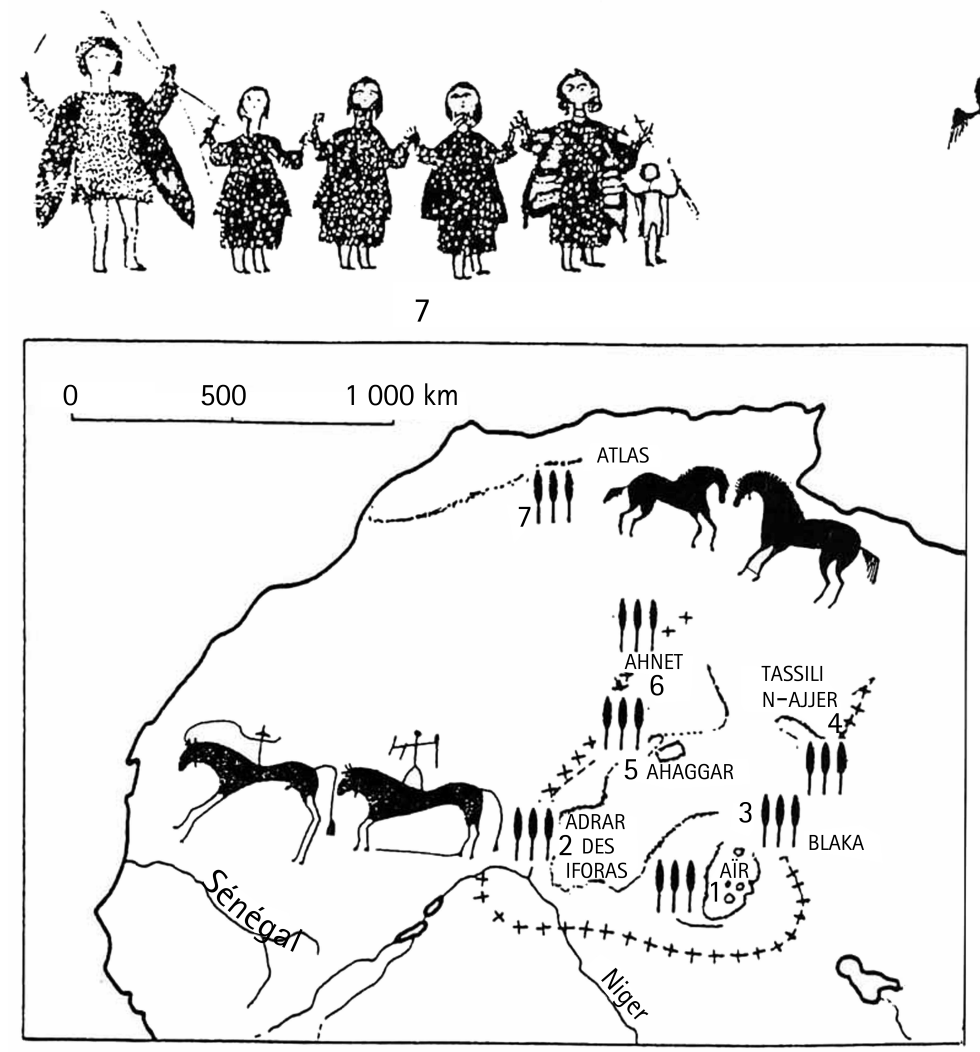

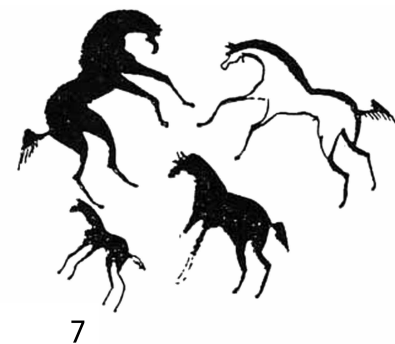

Fig. 5 - Répartition géographique des représentations de guerriers armés de plusieurs lances ou javelots recouvrant la majeure partie du domaine touareg $(++++)$, laquelle est identique à celle des chevaux du style levretté (1 - Téloues; 2 - Déladjou; 7 - Stèles funéraires peintes de Djorf Torba)

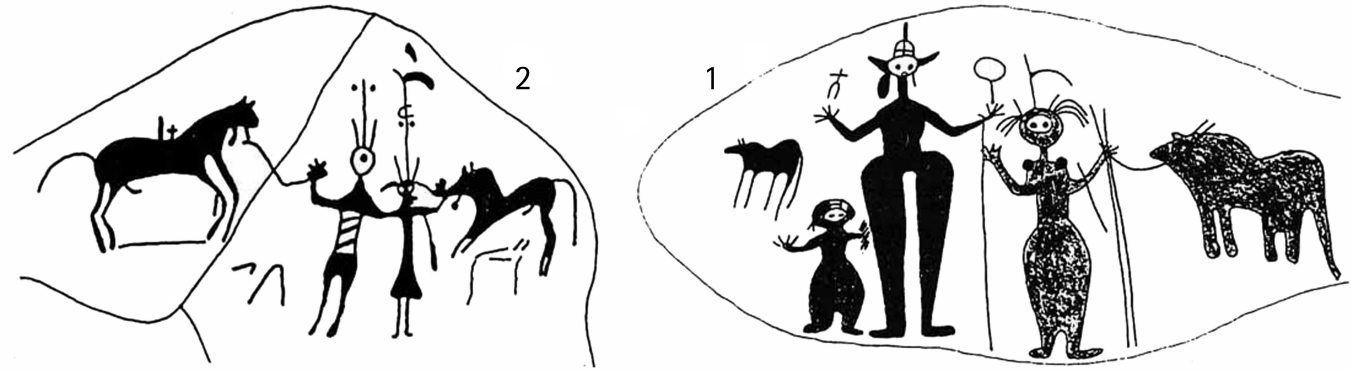

rique. La troisième tombe, de même architecture que la précédente, est sensiblement contemporaine des deux autres par le bol en terre cuite entièrement décoré de triangles incisés et muni de deux anses latérales raccordées à une embase creuse retrouvé dans la chambre funéraire : par sa forme et son décor géométrique, ce vase s'apparente aux poteries retrouvées dans des tombeaux datés du $\mathrm{IV}^{\mathrm{e}}$ siècle après J.-C. situés plus au nord, à Abalessa dans l'Ahaggar et à Germa au Fezzan méridional (Camps 1974). Des poteries peintes de motifs géométriques ont été exhumées de différents sites de la moyenne vallée du Niger datés des $\mathrm{V}^{\mathrm{e}}$-IX ${ }^{\mathrm{e}}$ siècles après J.-C. Le gisement archéologique de Djenne-Jeno a livré, en outre, deux perles en verre d'époque romaine (McIntosh 1994). Un peu plus au sud, à Kissi (nord-est du Burkina-Faso), les fouilles de sépultures datées de cette même époque ont mis au jour des bijoux en laiton, des poignards, des épées, des tissus et des perles en verre témoignant aussi d'influences nord-africaines et d'échanges avec le monde berbère (Magnavita 2009). La transformation des mythes et des croyances antéislamiques consécutive à l'adoption de la religion musulmane a conduit les Touaregs à abandonner leur tradition d'art rupestre dans le courant du II millénaire après J.-C.

\section{Perspectives de recherche}

Les quelques points développés ci-dessus montrent combien les apports de l'art rupestre du Sahara méridional sont complémentaires de ceux fournis par les autres domaines de l'archéologie. En étendant la méthodologie mise en œuvre au sud-ouest de l'Aïr et au nord-ouest de l'Adrar des Iforas à des secteurs encore peu explorés, certaines des hypothèses avancées ci-dessus pour les huit derniers millénaires d'histoire du 
Pour le sommaire complet ou la commande du numéro cliquez ici: www.nda.msh-paris.fr

\section{Christian Dupuy | Les apports archéologiques des gravures rupestres de l'Aïr et de l'Adrar des Iforas}

peuplement de l'Afrique septentrionale seront probablement confirmées, d'autres peut-être nuancées et, selon toute vraisemblance, de nouvelles pistes de réflexions seront ouvertes. Les recherches à venir ne pourront faire l'économie de fouilles au voisinage des stations d'art rupestre comme cela a été fait à Iwelen dans l'Aïr au début des années 1980. De telles recherches supposent des moyens humains et matériels et des financements sur plusieurs années, très difficiles à obtenir aujourd'hui...

\section{Bibliographie}

Abelanet, J. 1986. Signes sans paroles. Cent siècles d'art rupestre en Europe occidentale. Paris, Hachette, 345 pages.

Amblard-PISON, S. 2006. Communautés villageoises néolithiques des Dhars Tichitt et Oualata (Mauritanie). Oxford, J. \&t Hedges (BAR International series, 1546), 351 pages.

BEDAUX, R.M.A. 1972. "Tellem, reconnaissance archéologique d'une culture de l'Ouest africain au Moyen Âge : recherches architectoniques", Journal de la Société des africanistes, 42 : 103-185.

Bedaux, R. M. A., T.S. Constandse-Westermann, L. Hacouebord, A. G. Lange \& J.D. VAN DER WAALS. 1978. "Recherches archéologiques dans le delta intérieur du Niger (Mali)", Palaeohistoria, $20: 91-220$.

Bouller, C., A. Person, J.-F. Sallege \& J. Polet. 2002-2003. "Bilan chronologique de la culture Nok et nouvelles datations sur des sculptures", Afrique, Archéologie et Art, 2 : 9-28.

CAMPS, G. 1974. "L'âge du tombeau de Tin-Hinan, ancêtre des Touaregs du Hoggar ", Zéphyrus, XXV : 497-516.

CAmps, G. 1995. "Djorf Torba", in: G. Camps (éd.), Encyclopédie Berbère, XVI, Djalut-Dougga. Aix-en-Provence, Édisud : 2477-2488.

CHenORKIAN, R. 1988. Les armes métalliques dans l'art protohistorique de I'Occident méditerranéen. Marseille, CNRS Éditions, 414 pages.

DupuY, C. 1988. "Évolution iconographique de trois stations de gravures rupestres de l'Aïr méridional (Niger)", Cahiers ORSTOM, série Sciences humaines, 24, $2: 303-315$.

Dupuy, C. 1991. "Les gravures rupestres de I'Adrar des Iforas dans le contexte de l'art saharien: une contribution à I'histoire du peuplement pastoral en Afrique septentrionale du Néolithique à nos jours", Aix-en-Provence, université de Provence, thèse de doctorat, 2 tomes, 404 pages.

Dupuy, C. 1994. "Signes gravés au Sahara en contexte animalier et les débuts de la métallurgie ouest-africaine", Préhistoire et Anthropologie méditerranéennes, 3 : 103-124.

Dupuy, C. 1999. "L'art rupestre à gravures naturalistes de l'Adrar des Iforas (Mali)", Sahara, 11 : 69-86.
DupuY, C. 2005. "Les gravures de bœufs à bosse de l'Aïr (Niger) et de I'Adrar des Iforas (Mali)», Bulletin de la Société d'études et de recherches préhistoriques des Eyzies, 54 : 63-90.

Dupur, C. 2006. "L'Adrar des Iforas à l'époque des chars : art, religion, rapports sociaux et relations à grande distance", Sahara, $17: 29-50$.

LHOTE, H. 1972. Les gravures rupestres du nord-ouest de I'Air. Paris, AMG, 205 pages.

LHOTE, H. 1979. Les gravures rupestres de l'oued Mammanet (nord-ouest du massif de l'Airr). Paris, Les Nouvelles Éditions africaines, 431 pages.

LHOTE, H. 1987. Les gravures du pourtour occidental et du centre de l'Aïr. Paris, Éditions Recherches sur les civilisations (Mémoire, 70), 281 pages.

Magnavita, S., P. Breunig, D. Ishaya \& O. Adebayo. 2009. "Iron Age beginnings at the southwestern margins of Lake Chad", in : S. Magnavita, L. Kote, P. Breunig \& O.A. Ide (éd.), Crossroads. Cultural and Technological Developments in First Millenium BC/ $A D$ West Africa. Francfort, Africa Magna Verlag (Journal of African Archaelogy, Monograph series, 2) : 27-58.

MagnaVITA, S. 2009. "Sahelian Crossroads : some aspects on the Iron Age sites of Kissi, Burkina Faso", in : S. Magnavita, L. Kote, P. Breunig \&t O.A. IDE (éd.), Crossroads. Cultural and Technological Developments in First Millenium BC/AD West Africa. Francfort, Africa Magna Verlag (Journal of African Archaelogy, Monograph series, 2) : 79-104.

McIntosH, S. K. (éd.). 1994. Excavations at Jenné-Jeno, Hambarketolo and Kaniana (Inland Niger Delta, Mali), the 1981 Season. Berkeley - Los Angeles, University of California Press (University of California publications in anthropology, 20), 605 pages.

McIntosh, S. K. \& R.J. Mclntosh. 1980. Prehistoric Investigations in the Region of Jenne, Mali. A Study in the Developpment of Urbanism in the Sahel. Oxford, BAR (Cambridge Monographs in African Archaeology, 2 ; BAR International series, 89), 541 pages.

PARIS, F. 1990. "Les sépultures monumentales d'Iwelen", Journal des africanistes, 60, $1:$ 44-74.

PARIS, F. 1996. Les sépultures du Sahara nigérien du Néolithique à I'Is/amisation. Paris, Orstom (Études et Thèses), 2 tomes, 621 pages.

Rodrigue, A., 1999. L'art rupestre du Haut Atlas marocain. Paris, L'Harmattan, 420 pages.

Roset, J.-P. 2007. "La culture d'Iwelen et les débuts de la métallurgie du cuivre dans l'Aïr, au Nigern, in : J. GUILAINE (éd.), Le Chalcolithique et la construction des inégalités. II, Proche et Moyen-Orient, Amérique, Afrique. Paris, Errance : 107-136.

Rupp, N., J. Ameje \& P. Breunig. 2005. "New studies on the Nok culture of Central Nigeria ", Journal of African Archaelogy, 3, 2 : 283-290.

Seignobos, C., H. Tourneux, A. Hentic \& D. Planchenault. 1987. Le poney $d u$ Logone et les derniers peuples cavaliers. Essai d'approche historique. Maison-Alfort, Institut d'élevage et de médecine vétérinaire des pays tropicaux (Études et synthèses de l'IEMVT, 23), 213 pages. 